\title{
ANÁLISIS SOCIO ECONÓMICO Y CARBONO ALMACENADO EN SISTEMAS AGROFORESTALES DE CACAO (Theobroma cacao L.) EN HUÁNUCO
}

\section{SOCIOECONOMIC ANALYSIS AND CARBON STOCK IN AGROFORESTRY SYSTEMS WITH COCOA (Theobroma cacao L.) IN HUANUCO}

\author{
Vicente S. Pocomucha ${ }^{1}$, Julio Alegre ${ }^{2}$ y Luís Abregú ${ }^{3}$
}

\begin{abstract}
Resumen
Se evaluó el carbono almacenado en los sistemas agroforestales de cacao (Theobroma cacao L.) en el distrito José Crespo y Castillo (Huánuco, Perú). El contenido total de carbono se interrelacionó con los factores sociales, económicos y ambientales de las parcelas y familias que lo cultivan. La investigación se realizó mediante cuestionarios tipo Likert encuestando a 36 productores pertenecientes a la Asociación de Productores Agropecuarios. La estimación de carbono se realizó mediante la metodología planteada por Arévalo et al. (2003), evaluándose la biomasa aérea y carbono en el suelo. Los cacaotales tuvieron un dosel de sombra simple con un promedio de cuatro especies a razón de 69 árboles ha $^{-1}$. El valor promedio total de carbono acumulado en los cacaotales fue $131.18 \mathrm{t} \mathrm{C}^{-1}$ (65.61 t C ha-1 para biomasa aérea y $65.57 \mathrm{t} \mathrm{C} \mathrm{ha-1}$ para el componente suelo). El manejo de las parcelas en su mayoría es de subsistencia y los rendimientos del cacao son bajos (489 $\left.\mathrm{kg} \mathrm{ha}^{-1}\right)$. Los productores desde el enfoque social, muestran actitudes positivas de satisfacción en la aplicación del SAF de cacao (67\%), la intención de continuidad (72\%), motivación por la agricultura (47\%), trabajar con la familia (56\%) y en el enfoque económico reflejan dedicarse a los SAF de cacao (56\%). Los productores cacaoteros, no reciben ningún beneficio por servicios ambientales, manejando sus parcelas de cacao, solamente obtienen frutos, semillas, madera y leña.
\end{abstract}

Palabras clave: Biomasa, sistemas agroforestales, factores sociales, económicos, ambientales, Likert.

\begin{abstract}
The study was conducted in the district of Jose Crespo y Castillo (Huanuco, Peru) to evaluate carbon stocks in agroforestry systems with cocoa (Theobroma cacao L.). Total content of carbon was interrelated with social, economic and environmental factors of the families that produce cocoa. The research was carried out by applying a Likert questionnaire.to a representative sample of 36 cocoa producers belonging to the Association of Agricultural Producers. Carbon estimation was performed by measuring biomass and soil carbon using the methodology developed by Arevalo et al. (2003). The cocoa trees are diversified into four different simple shade trees with 69 trees $\mathrm{ha}^{-1}$. The total average carbon accumulated in the cocoa systems was $131.18 \mathrm{t} \mathrm{C} \mathrm{ha-1}(65.61 \mathrm{t}$ $\mathrm{C} \mathrm{ha}^{-1}$ aerial biomass and $65.57 \mathrm{t} \mathrm{C} \mathrm{ha}^{-1}$ for the soil). The plot management is mainly for subsistence and cocoa yields are low $\left(489 \mathrm{~kg} \mathrm{ha}^{-1}\right)$. From the social aspects the producers show positive satisfaction attitudes in implementing the ASC (67\%); in the intention of continuity (72\%), motivation for agriculture (47\%), working with the family (56\%) and focus on the ASC (56\%). The cocoa producers receive no benefit for environmental services to manage their cocoa plots, they only get fruits, seeds, wood and firewood.
\end{abstract}

Key words: Biomass, agroforestry systems, social, economic, and environmental factors, Likert.

\section{Introducción.}

El cambio climático en el mundo, está causando preocupación en la sociedad, actualmente existe un consenso en la comunidad científica que es indispensable reducir las emisiones de gases de efecto invernadero (GEI) de la atmósfera con acciones efectivas (Torres et al., 2008). La deforestación y degradación de bosques tropicales adicionan cerca del
$20 \%$ (1.6 billones de $\mathrm{t} \mathrm{C}$ año ${ }^{-1}$ ) del total de las emisiones de carbono a la atmosfera a nivel mundial (Parker et al., 2009). A este contexto la deforestación en el Perú aporta con el $47 \%$ de $\mathrm{CO}_{2}$ causada por la agricultura y ganadería migratoria, establecida por métodos ancestrales de tumba, roza, quema, débil sistema de tenencia de tierras, cultivos ilícitos y uso de leña (Tavera, 2015). 
Los sistemas agroforestales (SAF), son considerados como una opción que pueden almacenar entre 12 y 228 t C ha-1 (Alegre et al., 2001; Murty et al., 2013) a fin de poder atenuar los graves efectos que producirá el cambio climático. La importancia de estos sistemas se incrementa cuando se asocian con especies forestales (maderables, frutales e industriales) que presentan mayor eficiencia en la fijación y almacenamiento de carbono (Somarriba \& Harvey, 2003; Concha et al., 2007). Esto tiene un impacto positivo asociado a la conservación de la biodiversidad, mitigación de la pobreza y seguridad alimentaria ya que funcionan a través de múltiples interacciones entre árboles, cultivos, animales, ambiente y el ser humano, así como en la reducción de costos de producción (Beer et al., 2004).

En la región de Huánuco, el cultivo de cacao es considerado como uno de los productos agrícolas no tradicionales de importancia social y económica, por generar ingresos económicos en las familias, empleo de mano de obra familiar (5 miembros) que poseen en promedio 6.5 ha de terreno (MINAG, 2005; Ríos et al., 2008; Valencia et al., 2007). El cacao se viene cultivando en asociación con especies forestales en áreas menores a 2 ha (MINAG, 2003), se está manejando empleando técnicas agrícolas de subsistencia; donde los productores cacaoteros pertenecen a alguna asociación, comité o cooperativa, otros trabajan independientemente; obteniendo rendimientos menores al promedio nacional (510 kg $\mathrm{ha}^{-1}$ ) y a nivel de la zona de estudio que varía entre 300 y $400 \mathrm{~kg} \mathrm{ha}^{-1}$ (Arévalo et al., 2004; MINAG, 2005).

Los SAF de cacao producen muchas ventajas positivas, siendo necesario caracterizar los factores más relevantes en el aspecto socio económico y ambiental, para mejorar las potencialidades de los cacaotales y promoverse como una alternativa de manejo sostenible (Roa et al., 2009). El objetivo principal de este estudio fue evaluar el carbono almacenado en los sistemas agroforestales de cacao interrelacionados con los factores sociales, económicos y ambientales de los cacaoteros en el distrito José Crespo y Castillo en Huánuco, Perú.

\section{Materiales y métodos.}

El estudio se realizó en la margen derecha del río Huallaga, distrito José Crespo y Castillo, provincia Leoncio Prado, región Huánuco. Ubicado a $08^{\circ} 21^{\prime}$ 47.7” de Latitud Sur y 76 $10^{\prime} 16^{\prime}$ ' de Longitud Oeste, a una altitud $600 \mathrm{msnm}$, con una superficie de 108353 ha (Figura 1). El clima es tropical húmedo, la temperatura media anual oscila entre $24-31^{\circ} \mathrm{C}$ y precipitación anual promedio de $2400 \mathrm{~mm}$.
La población objetivo del estudio, estuvo conformada por 174 productores cacaoteros registrados en la Asociación de Productores Agropecuarios de la provincia de Leoncio Prado, región Huánuco del Ministerio de Agricultura. Se trabajó con una muestra aleatoria de 36 productores (n=36) según criterio de Hernández et al. (2003).

La recopilación de información, relacionada a los componentes sociales, económicos y ambientales, se realizó en cada uno de los 36 cacaotales, mediante un cuestionario del tipo Likert, donde los puntajes y alternativas fueron: $5=$ muy de acuerdo, $4=$ de acuerdo, 3 = ni de acuerdo, ni en desacuerdo, 2 = en desacuerdo y 1 = muy en desacuerdo. Se estableció una categoría cuantitativa equivalente de: 4-5 puntos (afirmación/aceptación a la pregunta y/o respuesta), 3 puntos (afirmación con tendencia al rechazo o negación), 1-2 puntos (negación o rechazo a la pregunta $\mathrm{y} / \mathrm{o}$ respuesta). Para mantener la confiabilidad de las respuestas, las entrevistas se realizaron en horas de la mañana, previamente se motivaron a los productores cacaoteros, en coordinación con las autoridades locales (Tenientes alcaldes y/o gobernadores).

Para la estimación de carbono almacenado en biomasa vegetal total y carbono en el suelo de los sistemas agroforestales de cacao, se utilizó la metodología desarrollada por Arévalo et al. (2003) siendo:

- Carbono en la biomasa vegetal total (CBV) CBV $\left(\mathrm{t} \mathrm{ha}^{-1}\right)=\mathrm{BVT}^{*} 0.45$

Donde:

$\mathrm{CBV}=$ Carbono en la biomasa vegetal $\left(\mathrm{t} \mathrm{ha}^{-1}\right)$

$\mathrm{BVT}=$ Biomasa vegetal total

$0.45=$ Constante

- Carbono en el suelo (CS)

Donde: $\mathrm{CS}\left(\mathrm{t} \mathrm{ha}^{-1}\right)=(\mathrm{PVs} * \% \mathrm{C}) / 100$

$\mathrm{CS}=$ Carbono en el suelo $\left(\mathrm{t} \mathrm{ha} \mathrm{h}^{-1}\right)$

$\mathrm{PVs}=$ Peso del volumen de suelo

$\% \mathrm{C}=$ Resultados de carbono (\%) analizados en el laboratorio

100 = Factor de conversión

Las muestras se tomaron en forma aleatoria, utilizándose como unidad de muestreo parcelas de $4 \mathrm{~m}$ x 25 m y 5 m x 100 m; evaluándose para el cacao el diámetro basal a $30 \mathrm{~cm}$ desde el suelo (Andrade, 2003) y en el componente arbóreo a $1.3 \mathrm{~m}$ a la altura del pecho (dap). El análisis de la información se realizó mediante la estadística descriptiva.

\section{Resultados y discusión.}

Tipificación del productor y sistemas agroforestales de cacao. 


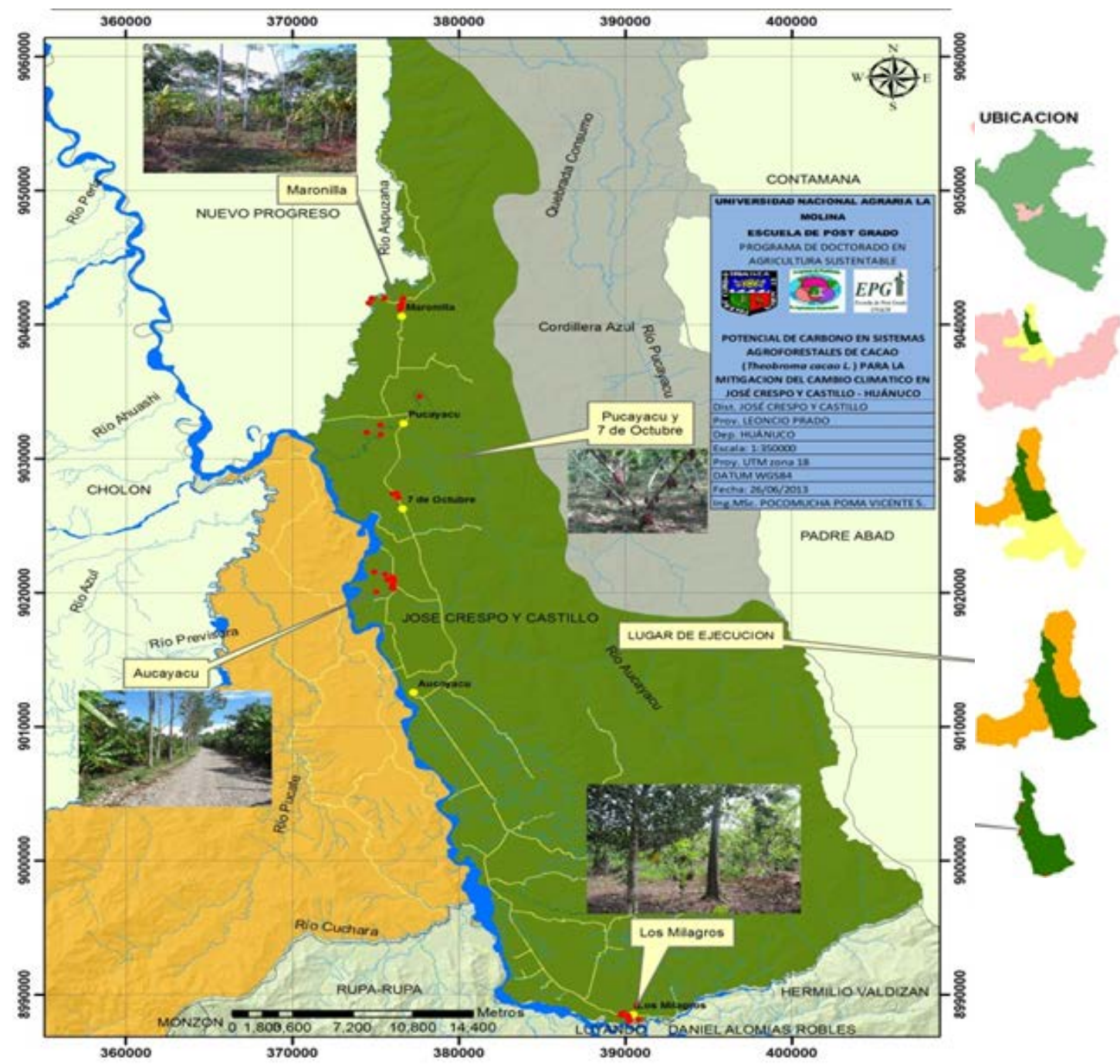

Figura 1. Mapa de ubicación de la zona de estudio. Distrito José Crespo y Castillo, en Huánuco, Perú.

El 86.1\% de productores son varones y fue notoria la poca participación de las mujeres en la población rural $(13.9 \%)$ económicamente activa. El grado de educación predominante fue el nivel primario (69.4\%). Los productores en su mayoría fueron migrantes de varios sitios del país, el $47.2 \%$ procede de la sierra, el $41.7 \%$ de la selva y el $11.1 \%$ de la costa (Tabla 1). Estos resultados nos muestran para la zona de estudio, los productores cacaoteros son migrantes y tienen un arraigo cultural de trabajo en el campo, conocen de la agricultura que facilita la implementación y establecimiento de sistemas agroforestales de cacao; a pesar de provenir de la sierra o la costa y no tener experiencia en cultivos tropicales como el cacao. En el desarrollo de diferentes sistemas de uso de tierras en el Perú, las sociedades locales no son estáticas, la mayoría de las zonas rurales enfrentan un crecimiento poblacional, inmigraciones y modificaciones en la agricultura (Wiersum, 1985).
En relación a la posesión de tierras el 66.7\% declaró tener título de propiedad y el 33.3\% no son propietarios (Tabla 1). Estos últimos no pueden realizar trámites legales, no tienen acceso a financiamiento para obtener créditos bancarios, créditos hipotecarios, créditos financieros y seguros agrarios. Este escenario es característica común en la Amazonía peruana (Vásquez et al., 2008; Vega, 2012), en tanto, la estructura de las propiedades se encuentra altamente fragmentada. La producción de cacao ocurre en pequeñas parcelas, sobre tierras soslayadas (De Janvry, 1981).

La edad de los productores cacaoteros presentó un rango entre 36 y 74 años y un promedio de 56 años (Tabla 2). Con la edad avanzada los productores poseen mayor conocimiento en el manejo del cultivo de cacao asociado con especies forestales. Este factor estaría afectando la producción de cacao, puesto que en el manejo del cultivo, se requiere de mayor esfuerzo físico, ya que en gran parte los trabajos de 
Tabla 1. Variables sexo, grado de instrucción, lugar de procedencia y tenencia de tierra de los productores cacaoteros. Distrito José Crespo y Castillo.

\begin{tabular}{lc}
\hline Variables & Porcentaje (\%) \\
\hline SEXO & 86.1 \\
Masculino & 13.9 \\
Femenino & 69.4 \\
GRADO DE INSTRUCCIÓN & 19.4 \\
Primaria & 8.3 \\
Secundaria & 2.8 \\
Instituto & \\
Universidad & 47.2 \\
LUGAR DE PROCEDENCIA & 41.7 \\
Sierra & 11.1 \\
Selva & \\
Costa & 66.7 \\
TENENCIA DE TIERRA & 27.8 \\
Título de propiedad & 2.8 \\
Posesión con certificado & 2.8 \\
Posesión sin certificado & \\
Arrendado &
\end{tabular}

campo son de forma manual. El número de miembros por familia dedicados al SAF de cacao en promedio fue de tres personas; así como lo refiere Altieri (1999), este componente de mano de obra y tamaño de familia, son factores críticos para la comprensión lógica de un sistema agroforestal. Para generar mayor rentabilidad económica, deben involucrarse mayor número de miembros de familia a las labores productivas del cacao (Adejumo, 2005; Bentes et al., 2005).

Caracterización de factores cualitativos.

a)Ambiental

Aceptabilidad del cacao CCN-51: Del total de agricultores entrevistados, 30 productores cacaoteros (88.9\%) mostraron alto grado de aceptación (Figura 2), vienen cultivando el clon CCN-51 de cacao, de procedencia ecuatoriana, por presentar características deseables de fácil manejo (árbol pequeño, mayor rendimiento e inicio de producción a los dos años), utilizando distanciamientos de $3 \mathrm{~m}$ x $3 \mathrm{~m}$ entre plantas de cacao y las especies forestales como sostienen Vásquez et al. (2008) y Carrión (2012). La actividad cacaotera presenta problemas de enfermedades, manejo de sombra, manejo de malezas, manejo de poda, limitado uso de variedades mejoradas, créditos para el manejo del cultivo, comercialización, poca extensión de terrenos y productores de escasos recursos (Guiltinan \& Maxinova, 2002).

Asociación entre aire limpio vs contaminación ambiental: La totalidad de productores opinan que el aire limpio es indicativo de la ausencia de contaminación, considerando al árbol un componente principal del SAF que almacena carbono, haciéndolo potencialmente sostenible, especialmente si son considerados en proyectos de bonos de carbono (Figura 2). También por la producción de oxígeno se genera rentabilidad económica y contribuye en la reducción de riesgos de vulnerabilidad de las parcelas Dagang \& Nair (2003).

\section{b)Social}

Grado de mejora en las parcelas SAF: En el mantenimiento e incremento de la productividad de cultivos, con las capacitaciones (80.6\%) declararon haber obtenido mejoras en sus parcelas de SAF de cacao, al utilizar variedades mejoradas de cacao e incluir especies forestales. Esto demuestra que los efectos de las capacitaciones fueron positivas para mejorar el manejo de las parcelas, aún en condiciones ecológicas, económicas y sociales inestables (Taylor \& Beniest, 2006).

Importancia del trabajo familiar: Las labores de campo son realizadas con la mano de obra familiar, lo cual es considerado como un factor importante para el $86.2 \%$ de los entrevistados, lo que les permite tener estabilidad en la familia y generar ingresos económicos (Ríos et al., 2003; Mendieta \& Rocha, 2007).

Grado de satisfacción y motivación por el SAF de cacao: El $100 \%$ y el $88.9 \%$ de los productores se encuentran satisfechos con alta motivación (Figura 3), ya que los SAF de cacao son formas de uso de la tierra, que satisfacen necesidades, económicas, ambientales y sociales (Renda et al., 1997), por asociarse con especies forestales $y$ frutales que diversifican la producción e incrementan los beneficios, económicos, ambientales y sociales de los
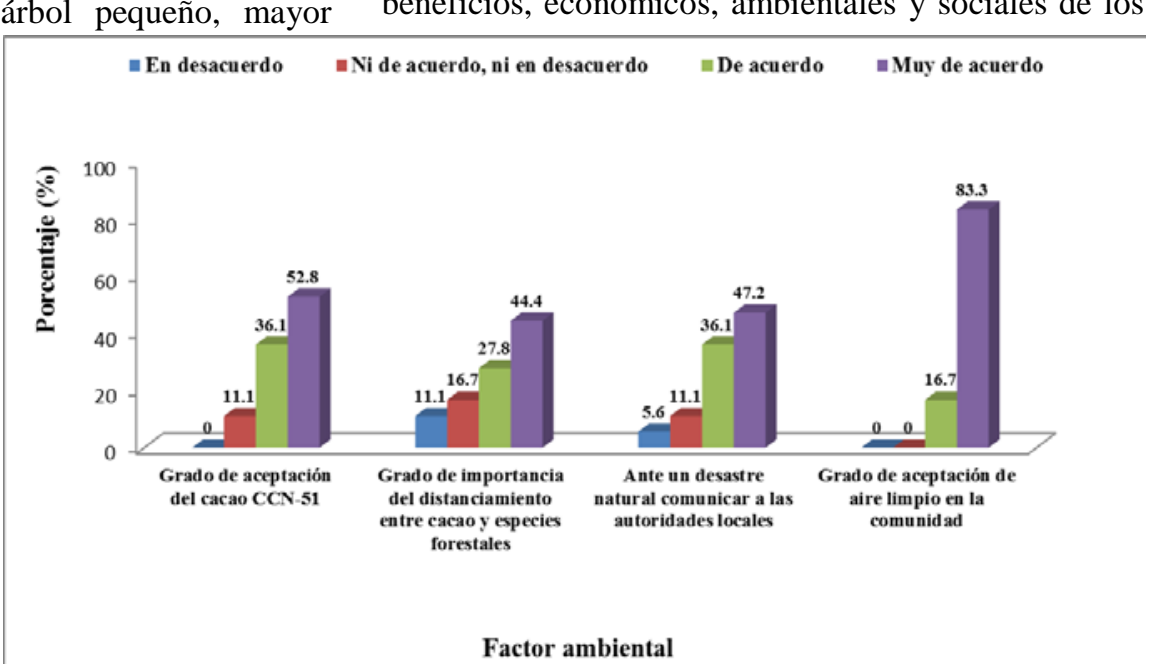

Figura 2. Actitudes de los productores cacaoteros, respecto a variables ambientales. Distrito José Crespo y Castillo. 


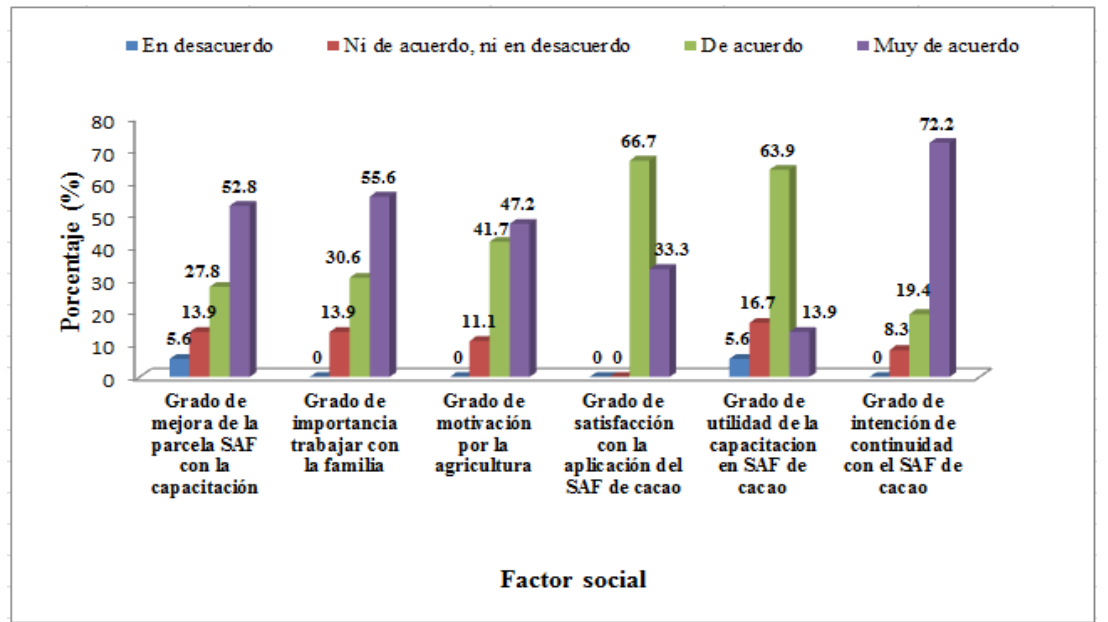

Figura 3. Actitudes de los productores cacaoteros, respecto a variables sociales. Distrito José Crespo y Castillo.

productores cacaoteros.

\section{Económico}

Ventajas por dedicación a las parcelas SAF: El $86.2 \%$ de los encuestados señaló muy importante la dedicación a los SAF. Respecto a la obtención de productos, el $100 \%$ de productores opinan un mayor ingreso porque les permite obtener simultáneamente todo el año diferentes productos como madera, leña, frutos y semillas. La totalidad de productores están dispuestos a negociar los pagos por bonos de carbono al manejar $\mathrm{y}$ ofrecer servicios ambientales por almacenamiento de carbono en sus parcelas SAF de cacao (Figura 4) así como lo referencian Nair (1983); Villanueva \& Wachtel (2009).

Caracterización de factores cuantitativos.

a)Ambiental

Asociación con especies forestales: El tiempo promedio de producción de los SAF de cacao fue de siete años con un máximo de 25 años. Últimamente el cultivo es considerado como promisorio y está siendo kg día-1) (Heising, 2006).

\section{b)Social}

adoptado como sistemas agroforestales asociando de uno a ocho especies forestales especialmente con guaba (Inga edulis L.), bolaina (Guazuma crinita C. Martius), capirona (Calycophyllum spruceanum (Benth) Hook F. Ex), con una densidad promedio de 69 árboles $\mathrm{ha}^{-1}$ coincidiendo con los resultados referidos por Vásquez et al. (2008); Villalobos \& Acuña (2009). Dentro de un sistema SAF, el árbol es importante ya que producen interacciones ecológicas y económicas entre los componentes arbóreos y no arbóreos que incrementan la diversidad biológica (Nair, 1983; Beer et al., 2004).

El área para el uso de otros cultivos en promedio fue 2.5 ha, con bosque primario y secundario con un máximo de 20 y 40 ha respectivamente (Tabla 2). El volumen promedio de leña utilizada fue de 65 $\mathrm{kg} / \mathrm{semana} /$ familia, resultando superior a la cantidad promedio de biomasa aprovechada a nivel nacional (6

Intensidad de capacitación y conocimiento sobre el SAF: Los agricultores llegaron a capacitarse como mínimo una hora y como máximo cinco horas alcanzando un promedio de tres horas de capacitación, tiempo no suficiente para recibir una buena capacitación y así obtener mejores rendimientos del cacao, especialmente en temas sobre las técnicas de manejo del cultivo que van desde la instalación hasta la poscosecha y que actualmente no están bien desarrolladas. El grado de mejora de la producción del cacao de acuerdo a la percepción de los productores

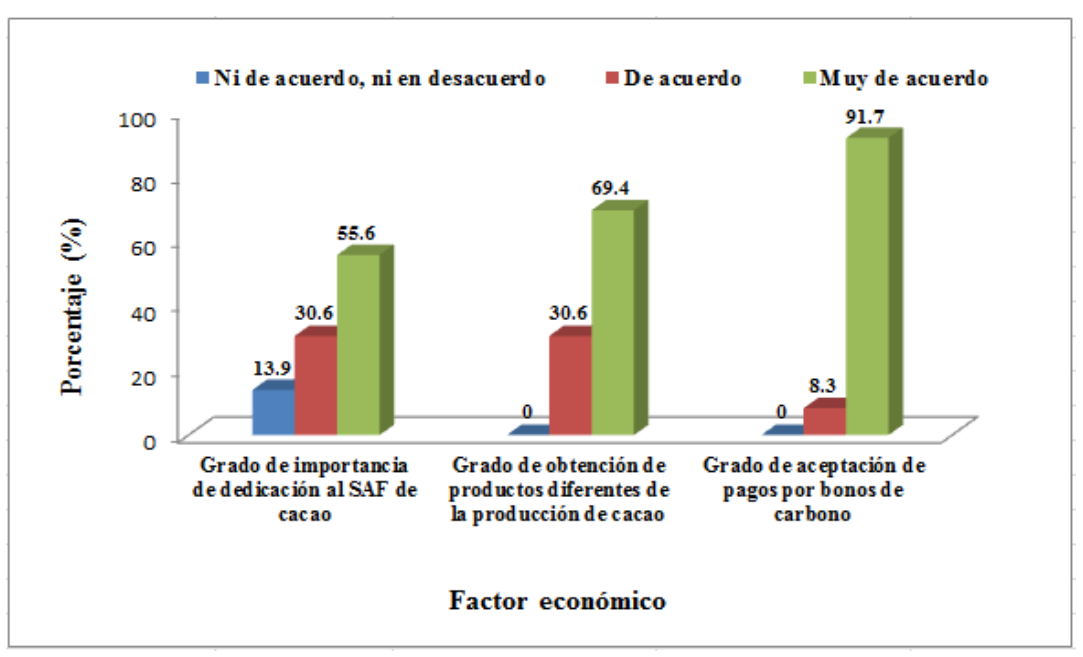

Figura 4. Actitudes de los productores cacaoteros, respecto a variables económicos. Distrito José Crespo y Castillo. está en función a la intensidad de capacitación recibida (Taylor \& Beniest, 2006). En el ámbito de estudio según las variables analizadas tiempo de conocimiento del SAF de cacao, los productores cacaoteros conocen el cultivo de cacao asociado en un promedio de 13 años, los agricultores de menor edad lo conocen desde hace tres años y los productores mayores de edad lo conocen desde hace 40 años (Tabla 2).

\section{Económicos}


Tabla 2. Estadística descriptiva para los factores cuantitativos ambiental, social y económico. Distrito José Crespo y Castillo.

\begin{tabular}{lcccc}
\hline Factores/Variables cuantitativas & Mínimo & Máximo & Promedio & $\begin{array}{c}\text { Desviación } \\
\text { estándar }\end{array}$ \\
\hline $\mathrm{N}^{\circ}$ de miembros de familia dedicados al SAF de cacao & 1 & 5 & 3 & 3.8 \\
Edad de los productores cacaoteros (años) & 36 & 74 & 56 & 11.46 \\
AMBIENTE & & & & \\
Total carbono almacenado (t/ha) & 71.93 & 216.31 & 131.18 & 63.18 \\
Carbono almacenado en biomasa aérea (t/ha) & 17.10 & 154.76 & 65.61 & 56.51 \\
Carbono almacenado en componente suelo (t/ha) & 49.35 & 99.59 & 65.57 & 18.68 \\
Número de especies asociadas con el cacao & 1.00 & 8.00 & 4.14 & 1.64 \\
Área de bosque primario (ha) & 0.00 & 40.00 & 3.63 & 8.61 \\
Área de bosque secundario (ha) & 0.00 & 20.00 & 3.07 & 4.74 \\
Cantidad de leña utilizada (Kg/mes) & 50.00 & 81.00 & 65.33 & 9.06 \\
SOCIAL & & & & \\
Hace que tiempo conoce el SAF de cacao (años) & 3.00 & 40.00 & 12.67 & 10.14 \\
Intensidad de capacitación (horas) & 1.00 & 5.00 & 3.00 & 0.92 \\
ECONOMICO & & & & \\
Rendimiento cacao (Kg/ha) & 100.00 & 1500.00 & 489.17 & 336.50 \\
Número de hectáreas a incrementar (ha) & 0.00 & 8.00 & 2.69 & 1.82 \\
\hline
\end{tabular}

Costos de producción y mantenimiento: Los resultados promedios encontrados de costos de producción (s/. 3 528.00/ha), de mantenimiento (s/. $379.00 / \mathrm{ha}$ ) y precio unitario (s/. 5.20/kg) son bajos. Estas variables inestables, dependen únicamente del ingreso por la venta del cacao, sin considerar los ingresos adicionales que generan las especies forestales. Además reflejan la inadecuada comercialización y el uso de tecnología convencional. El costo y mantenimiento de SAF de cacao, están relacionados a la tecnología utilizada (Torres et al., 2008).

El rendimiento promedio de cacao (489 $\left.\mathrm{kg} \mathrm{ha}^{-1}\right)$, registrado en la zona de estudio se encuentra por debajo del rendimiento nacional (510 $\mathrm{kg} \mathrm{ha}^{-1}$ ) (MINAG, 2005). Este resultado es debido a que el SAF de cacao es manejado en forma convencional, las especies forestales asociadas se encuentran dispersas en el área de cultivo y son producto de la regeneración natural concordando con (Ríos et al., 2003 y Vásquez et al., 2008).

El interés de los cacaoteros por incrementar las áreas con SAF de cacao, en promedio (3.0 ha) y máximo hasta (8.0 ha) (Tabla 2), no es factible por la carencia de áreas disponibles para la expansión de los SAF, excepto con productores que tienen bosque primario, secundario y otros cultivos. La conversión de bosque primario y secundario al cultivo de cacao, generaría mayores emisiones de $\mathrm{CO}_{2}$ a la atmosfera por lo que debe buscarse otras alternativas como, la recuperación de áreas de ganadería y suelos degradados mediante los SAF de cacao, la implementación de proyectos de pagos por bonos de carbono por conservar y manejar los diferentes tipos de bosques.

Carbono total almacenado en los SAF de cacao

El total de carbono almacenado en los SAF de cacao, en la biomasa aérea y el suelo presentó una variación desde 71.93 t C ha-1 hasta 216.31 t C ha $^{-1}$. Alcanzando un valor promedio total en los cacaotales de 131.18 t C ha-1 $^{-1} 65.61$ t C ha-1 $^{-1}$ para biomasa aérea y 65.57 t C ha $^{-1}$ para el componente suelo) (Tabla 2). Estos resultados se encuentran dentro del rango considerado por Alegre et al. (2001); Murty et al. (2013). La variación en la fijación y almacenamiento de carbono en los cacaotales, se incrementa cuando se asocian con especies maderables y frutales (Somarriba \& Harvey, 2003) y dependiendo del sistema de uso del suelo, mayormente están en función de la edad, diámetro, altura del componente forestal, tipos de especies, densidad de población de cada estrato y por comunidad (Concha et al., 2007).

\section{Conclusiones.} que:

En el presente trabajo de investigación se concluye

1. En el ámbito de estudio existe el conocimiento para el uso adecuado del potencial de carbono almacenado en sistemas agroforestales de cacao. Estos sistemas son diversificados y manejados principalmente por productores varones con educación de nivel primario, que superan los 50 años de edad. En su mayoría son migrantes de la sierra y más de la tercera parte no posee título de propiedad. Estos factores no son determinantes en el almacenamiento de carbono en los SAF de cacao, pero si generan limitaciones en gestiones de tipo social, económico, ambiental y legal para el manejo adecuado de los cacaotales.

2. Los factores socio económicos y ambientales evaluados presentan asociación con el potencial de carbono almacenado en los sistemas agroforestales de cacao del Distrito de José Crespo y Castillo. Mostraron una influencia significativa en el almacenamiento de carbono en los SAF de cacao. Lo que indica que existen productores cacaoteros que 
están muy de acuerdo en continuar con los SAF de cacao y que les gustaría estar incluidos en proyectos de mercado de carbono, así como se viene realizando desde hace mucho tiempo en otros países.

3. Desde el enfoque ambiental cabe resaltar que el $88.1 \%$ de los productores cacaoteros mostraron alto grado de aceptación al clon CCN-51 de cacao de fácil manejo, precoz, de porte pequeño, productivo, utilizando distanciamientos de $3 \mathrm{~m} \times 3 \mathrm{~m}$. Sin embargo, se viene manejando en forma convencional, obteniéndose bajos rendimientos comparado al promedio nacional.

4. Respecto a la percepción social se concluye que los productores cacaoteros en el área de estudio, son conscientes de la importancia del almacenamiento de carbono, que con sus saberes tradicionales dentro de su identidad cultural, hace que muestren actitudes positivas para desarrollar sistemas agroforestales de cacao integrales orientados al manejo sostenible de los recursos naturales.

5. Los cacaotales son asociados con árboles maderables y frutales de uno a ocho especies a razón de 69 árboles ha ${ }^{-1}$ como resultado de la regeneración natural, llegando a almacenar carbono en la biomasa aérea y el suelo, en promedio $131.18 \mathrm{t} \mathrm{C} \mathrm{ha}^{-1}$, siendo superior al promedio señalado para suelos tropicales húmedos de Sudamérica.

\section{Literatura citada.}

Adejumo T. 2005. Crop protection strategies for major diseases of cocoa, coffee and cashew in Nigeria. African Journal of Biotechnology. 4 (2): 143-150.

Alegre J., Arévalo L., Ricse A., Barbaran J. \& Palm C. 2001. Reservas de carbono y emisión de gases con diferentes sistemas de uso de la tierra en dos sitios de la Amazonía peruana. Symposium Internacional de Agroforestería. Manaus, Brasil EMBRAPA. 15 p.

Altieri M. 1999. Agroecología. Bases científicas para una agricultura sustentable. Editorial Nordan-Comunidad. Montevideo. 325 p.

Andrade, H. 2003. Estimación de captura de carbona en sistemas agroforestales. CATIE. Costa Rica. 104 p.

Arévalo E., Zúñiga L., Arévalo C. \& Adriazola J. 2004. Manejo integrado del cultivo y transferencia de tecnología en la Amazonia Peruana. Instituto de Cultivos Tropicales (ICT). Tarapoto - Perú. 184 p.

Arévalo L., Alegre J. \& Palm C. 2003. Manual de las reservas totales de carbono en los diferentes sistemas de uso de la tierra en Perú. Publicación de STC/CGIAR/Ministerio de agricultura. Pucallpa, Perú. $24 \mathrm{p}$.

Beer J., Ibrahim M., Somarriba E., Barrance A. \& Leakey R. 2004. Establecimiento y manejo de árboles en sistemas agroforestales. Árboles de Centroamérica. OFICATIE. $46 \mathrm{p}$.

Bentes M., Somarriba E., Pinto W. \& Pastrana A. 2005. Estado y manejo de nuevas plantaciones de cacao injertado en Alto Beni, Bolivia. Agroforestería en las Américas. N 43-44: 67-71.

Carrión J.S. 2012. Estudio de factibilidad para la producción y comercialización de cacao (Theobroma cacao L.) variedad CCN-51, Jama-Manabí. Quito, Tesis Ing. Agroempresas. Universidad San Francisco de Quito. 65 p.

Concha J.Y., Alegre J.C. \& Pocomucha V. 2007. Determinación de las reservas de carbono en la biomasa aérea de sistemas agroforestales de Theobroma cacao L. En el Departamento de San Martín, Perú. Ecología Aplicada. 6(1,2): 75-82.

Dagang A.B.K. \& Nair P.K.R. 2003. Silvopastoral research and adoption in Central America: recent findings and recommendations for future directions. Agroforestry Systems. 59: 149-155.

De Janvry A. 1981. The Agrarian Question and Reformism in LatinAmerica. Baltimore: Johns Hopkins Univ. Press. $328 \mathrm{p}$.

Guiltinan M. \& Maximova S. 2002. The Penn State Program in the molecular biology of Cacao.

Heising K. 2006. Diversidad de combustibles domésticos en el Perú y la falta de cocinas apropiadas. Lima, PE. Tresco-Andes. $12 \mathrm{p}$.

Hernández S.R., Fernández C.C. \& Baptista L.P. 2003. Metodología de la investigación. 3 ed. McGraw-Hill Interamericana eds, S.A. México. 705 p.

Mendieta L.M. \& Rocha M.L.R. 2007. Sistemas agroforestales. Universidad Nacional Agraria. Managua, Nicaragua. $117 \mathrm{p}$.

MINAG (Ministerio de Agricultura). 2005. Diagnostico cadena agroproductiva del cacao. $68 \mathrm{p}$.

2003. Caracterización de zonas productoras de cacao en el Perú y su competitividad. Programa para el desarrollo de la Amazonía. PROAMAZONAS. Lima, Perú. 221 p.

Murthy I., Gupta M., Tomar S., Munsi M., Tiwari R., Hegde G.T. \& Ravindranath N.H. 2013. Carbon sequestration potential of agroforestry systems in India. Centre for Sustainable Technologies, Indian Institute of Science, Bangalore, India. Earth Science \& Climatic Change. 4:1.

Nair P.K.R. 1983. Multiple land use and agroforestry. En: Nugent J. \& O’Connor M. (Eds). Better Crops for Food. CIBA Foundation Symposium 97. Londres: Pitman Books. p. 101-115.

Parker C., Mitchell A., Trivedi M. \& Mardas N. 2009. The Little REDD+ Book. Global Canopy Programme. 71 p.

Renda A., Calzadilla E., Jiménez M. \& Sánchez J. 1997. La agroforestería en Cuba. Red Latinoamericana de Cooperación Técnica en sistemas agroforestales. Oficina Regional de FAO para América Latina y el Caribe, Santiago de Chile. 64 p.

Rios J., Bastos J. \& Cordeiro A. 2008. Quantificação do carbono em sistemas de uso-da-terra no Distrito de José Crespo E Castillo, Peru. Archivos Latinoamericano de Producción animal. 16(3): 130-142.

Valencia F. \& Muñoz M. 2003. Expansión y trayectoria de la ganadería en la Amazonía: Alto Huallaga, Perú, UNAS Tingo María Perú. 133 p.

Roa H.A., Salgado M.G. \& Alvarez J. 2009. Análisis de la estructura arbórea del sistema agroforestal de cacao (Theobroma cacao L.) en el Soconusco, ChiapasMéxico. Acta biol. Colombia. 14(3): 97-110.

Somarriba E. \& Harvey C. 2003. Como integrar simultáneamente producción sostenible y conservación de la biodiversidad en cacaotales orgánicos indígenas? Agroforestería en las Américas 10 (37-38). 
Tavera J. 2015. Tecnología, cambio climático y el sector forestal. Pensamiento Crítico Vol. $20 \mathrm{~N}^{\circ}$ 1, pp. 145-154.

Taylor P. \& Beniest J. 2006. Diseño y capacitación en agroforestería. Herramientas para capacitadores. World Agroforestry Centre. 284 p.

Torres J., Tenorio A. \& Gómez A. 2008. Agroforestería: una estrategia de adaptación al cambio climático propuesta de adaptación tecnológica del cultivo de café y cacao en respuesta al cambio climático en San Martín. Ed. Soluciones Prácticas-ITDG. Lima, Perú. 124 p.

Valencia F., Fernández P., Ríos J. \& Muñoz M. 2007. Diagnóstico sectorial socioeconómico y ambiental. FLOAGRI. Tingo María, Perú. 168 p.

Vásquez R.W., Ríos J., Valencia F. \& Muñoz M. 2008 Caracterización de los sistemas agroforestales de la cuenca media margen derecha del río Huallaga Aucayacu - Perú. FLOAGRI. Tingo María, Perú. 81 p.
Vega M.E. 2012. El caso de la Cooperativa Agraria Industrial Naranjillo (COOPAIN): Expresión de biocomercio en el Perú. Tesis Mg. PUCP. Lima Perú. $136 \mathrm{p}$.

Villalobos M. \& Acuña K. 2009. Una experiencia de manejo ambiental en cacao en los territorios indígenas Bribri y Cabécar de Talamanca-Costa Rica. El caso del Proyecto Captura de Carbono. Turrialva, Costa Rica. 38 p.

Villanueva C. \& Wachtel G. 2009. Agroforestería en la región San Martín: manual de promoción y capacitación para extensionistas. Manual Técnico. 129 p.

Wiersum K. 1985. Significado de la organización social y de las actitudes culturales en el desarrollo forestal. Avances en la investigación agroforestal. Memoria de Seminario. Turrialba Costa Rica. p. 26-38.

${ }^{1}$ Graduando del programa de doctorado en Agricultura Sustentable, Universidad Nacional Agraria La Molina (UNALM), Perú. Profesor principal, Facultad de Recursos Naturales Renovables, Universidad Nacional Agraria de la Selva (UNAS). Tingo Maria, Huánuco (Perú). E-mail: vicente.pocomucha@unas.edu.pe.

2 Profesor principal, Facultad de Agronomía, Universidad Nacional Agraria La Molina (UNALM). Av. La Molina s/n La Molina, Lima, Perú. E-mail: jalegre@lamolina.edu.pe.

${ }^{3}$ Profesor asociado, Facultad de Ciencias Económicas y Administrativas, Universidad Nacional Agraria de la Selva (UNAS), Área de Desarrollo Humano, Huánuco (Perú). E-mail: luis.abregu@unas.edu.pe. 